\title{
Piezocision as a Novel Approach for Accelerating Orthodontic Treatment (Literature Review)
}

\author{
Yufridika, Nazruddin, Nurhayati Harahap \\ Department of Orthodontics \\ Faculty of Dentistry, Universitas Sumatera Utara \\ Medan, Indonesia
}

\begin{abstract}
According to the American Association of Orthodontists (AAO) the length of comprehensive orthodontic treatment can range from approximately 12 to 36 months, depending on treatment options and individual characteristics. One of the biggest challenges an adult orthodontic patient is the duration spent on treatment. A new, minimally invasive surgical technique in term of piezocision is developed to address this issue and reduce overall treatment time. Piezocision is a novel, minimally invasive technique combining micro-incisions and decortications made by a piezotome in order to enhance the rate of orthodontic tooth movement. Piezocision allows for rapid correction of severe malocclusions without the drawbacks of traumatic conventional corticotomy or any accelerated surgical procedures. Piezocision allows simultaneous hard and/or soft tissue grafting via selective tunneling to correct gingival recessions or bone deficiencies. Piezocision increases regional acceleratory phenomenon (RAP) is stated as osteopenia, a transient condition, giving a way to a complete remission of bone density gradually as physiological healing progress. During accelerating tooth movement, this approach increases the RANKL/OPG ratio. Combined with a proper treatment planning and a good understanding of the biological events involved, this novel technique can locally manipulate alveolar bone metabolism in order to obtain rapid and stable orthodontic results.
\end{abstract}

Keywords-piezocision, accelerated tooth movement, regional acceleratory phenomenon

\section{INTRODUCTION}

An increasing number of patients are demanding orthodontic treatment for improving esthetics and mastication system. Treatment duration is one of the concerned important challenges for both patients and orthodontists in treatment planning. Patients have specific demands and usually want to achieve their treatment goals as soon as possible to reduce the negative effects of orthodontic appliances in their social and professional lives. According to the American Association of Orthodontists (AAO), length of comprehensive orthodontic treatment can range from approximately 12 to 36 months, depending on treatment options and individual characteristics [1,2].

The rate of biological orthodontic tooth movement is approximately $1 \mathrm{~mm}$ over 4 weeks. Distalization of canines can take almost 7 months in cases of upper premolar extraction and maximum anchorage, leading to total treatment duration of 2 years. Various attempts were made to shorten timing needed for orthodontic tooth movement in facing this issue [1]. These attempts generally divided in three categories. First, a local or systemic administration of medicines such as prostaglandins, interleukins, leukotrienes, cyclic adenosine monophosphate, and vitamin $\mathrm{D}$ was done. Second category is mechanical or physical stimulation such as direct electrical current, pulsed electromagnetic field, samarium cobalt magnet, and low-energy laser. These two methods increase movement speed 0.3 to 1 times. The effectivity and side effects of medicines are not specifically well-known in the long term. Physical stimulation also has unwanted effects. The last category is oral surgery, including dental distraction, alveolar surgeries to undermine interseptal bone, and alveolar corticotomy, which have been used to correct malocclusions for over 100 years [2,3].

This literature review will describes the clinical application, examination of patient, advantage and disadvantage, molecular and cellular mechanism, surgical steps and case report of piezocision technique itself.

A. Clinical application

Piezocision can be used in a generalized, localized or sequential manner.

1. Generalized: If the correction of the malocclusion requires moving all of the teeth in both maxilla and mandible at the same time.

2. Localized: If the malocclusion affects only one part of the dentition or one arch (i.e. anterior crowding case with a perfect posterior occlusion, single tooth extrusions intrusions, etc.).

3. Sequential: If correction of the malocclusion requires a "staged" approach, selected areas or segment of the arch are being demineralized at different times during orthodontic treatment to achieve specific results $[4,5]$. 


\section{B. Initial periodontal examination and management}

A complete periodontal assessment including periodontal probing and full-mouth X-Ray must be conducted. Systematic scaling (and root planning if indicated) must be performed prior to the surgery. Any detected osseous lesion should be treated before conducting a piezocision procedure. It is highly recommend the use of preoperative three-dimensional imaging (Cone Beam Computerized Tomography) to locate areas of root proximity as well as the mental foramen. These images also allow the practitioner to assess the quantity and location of areas where a bone graft would be indicated [10].

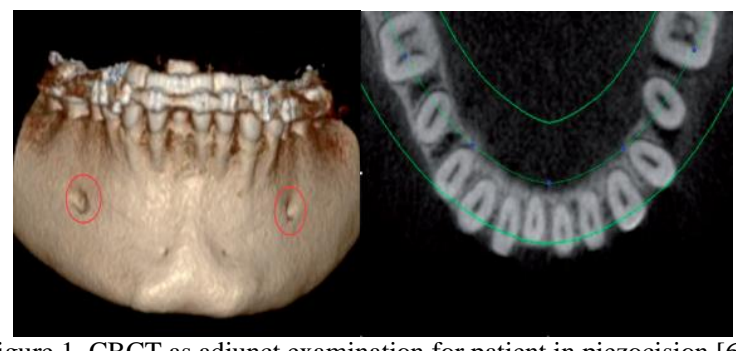

Figure 1. CBCT as adjunct examination for patient in piezocision [6].

\section{Indication}

Piezocision is indicated for dental type malocclusions as listed: Class I malocclusions with moderate to severe crowding (extraction and non extraction), selected Class II malocclusions (end-on), selected Class III malocclusions (dental), deep bite, open bite, distalization of molars, palatal version of root apices, rapid adult orthodontic treatment, orthodontic treatment combined with clear aligners, rapid intrusion and extrusion of teeth, prevention or simultaneous correction of osseous and mucogingival defects that may occur during or after orthodontic treatment, multidisciplinary treatments $[5,6]$

\section{Contraindications}

Patients are contraindicated if any medical history within such as medically compromised patients, patients on drugs modifying normal bone physiology (i.e. bisphosphonates), any bone pathology, ankylosed teeth, non-compliant patients, patient and/or operator having a pacemaker or any other active implant $[5,6]$.

\section{E. Advantages}

Piezocision is having similar clinical outcomes to those obtained by conventional corticotomy with the advantages of being shorter to perform, minimally invasive and much less traumatic for the patient. Piezocision can resolves majority of severe malocclusions within 5 to 9 months. Piezocision allows for an increase scope of treatment including allowing the correction of severe crowding without extraction when combining with bone graft. From a technical standpoints, a complete surgery on both the maxilla and mandible range from $45 \mathrm{~min}$ to $1 \mathrm{~h}$ with bone grafting against three to four hours for traditional techniques. There is not a report major edema or hematoma of the face as described after invasive corticotomy. Postoperative pain is usually minimal and well tolerated by patients $[6,7]$.

\section{F. Disadvantages and limitations of the technique}

Cortical incisions may present a risk of root damage particularly in areas of close root proximity because of the lack of muco-periosteal flap elevation. A risk also exists at the mental foramen. A panoramic radiograph and retroalveolar images of those areas are essential to the preparation of the surgery. Three-dimensional imaging can compensate for lacking direct vision of the bony structures. Extra care is also required as to the location of gingival incisions. It is very important to keep at least $2 \mathrm{~mm}$ from the gingival margin to avoid the formation of gingival cleft. Patients with ethnic gingival pigmentation, soft tissue incisions can create a cosmetic concern. In fact, incision may leave a trace without proper repigmentation, thereby causing cosmetic damages in patients with excessive gingival display. Those patients must be warned against potential risk of post-operative scars $[5,6]$.

\section{G. regional acceleratory phenomenon (rap)}

When the bone is injured, a very dynamic healing process occurs at the site of the bone injury. This biologic event is named as the regional acceleratory phenomenon (RAP), which is proportional to the extent of the surgical insult. There is a localized surge in osteoclastic and osteoblastic activities which results a decrease in bone density with an increased bone turnover in the early phase. The RAP begins within a few days after surgery, usually peaks in 1-2 months, and then slows down and disappears as remineralization sets in. Transient osteopenia after surgery was suggested to be responsible for the rapid tooth movement, as the teeth were now moving in a more 'pliable' environment. Cortical activation before orthodontic treatment induced the RAP to increase bone remodeling activity and tooth movement speed without any side effects $[1,5,7,10,11]$.

\section{H. Vibration in accelerated orthodontic tooth movement}

Vibration of a certain frequency, intensity, and duration can increase bone mass. Whole body vibration has been shown to significantly improve bone mineral density and structure. According to Nishimura et al, applying vibration on first molars in rats for 8 minutes once a week, resonance vibration $(60 \mathrm{~Hz})$ can increased tooth movement by $15 \%$ compared with the controls, stimulating more expression of RANKL and osteoclast formation in the PDL. ${ }^{9}$

\section{Molecular and cellular of accelerated orthodontic tooth movement}

Initial tooth movement is apparently rapid when applying orthodontic force after cortical activation and the cellular activity of periodontal cells such as fibroblasts, cementoblasts, osteoblasts, and osteoclasts 
was increased over a short time range. On the other hand, cellular activities of these cells are severely decreased after 6 months [11].

Methods that accelerate orthodontic tooth movement stimulate M-CSF and increase the RANKL/OPG ratio directly or indirectly through changes in blood flow and hypoxia, and tissue damage, promoting the production of cytokines including VEGF, TNF-a, interferon-b, ILs, matrix metalloproteinases, and others [8].

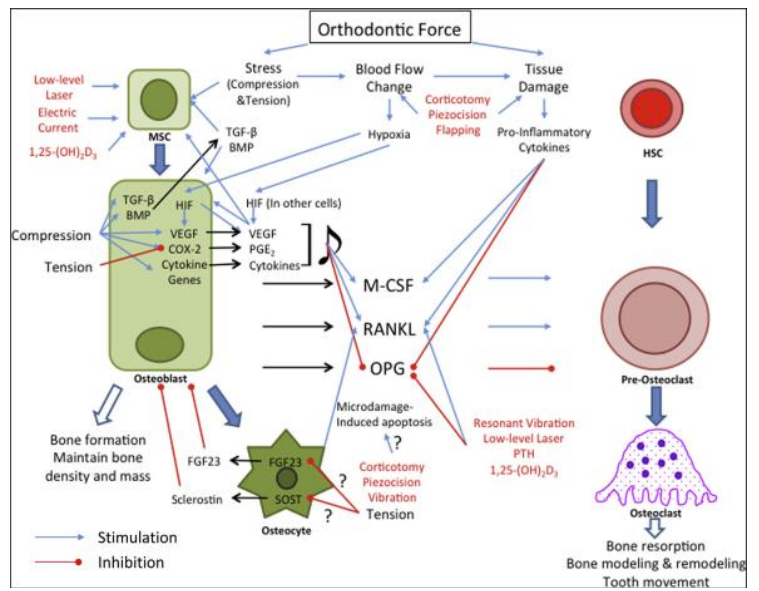

Figure 2. Methods to accelerate orthodontic tooth movement are shown in red. Blue arrow, Stimulation; red blunted arrow, inhibition; MSC, mesenchymal stem cell; HSC, hematopoietic stem cell; HIF, hypoxia inducible factor; FGF, fibroblast growth factor[8].

\section{DISCUSSION}

The surgery using Piezocision can be performed 1 week following placement of the fixed orthodontic appliance. Vertical interproximal incisions are made under local anesthesia, below the interdental papilla, on the buccal side of each jaw using a microsurgical blade or a blade No. 15. Incisions should be kept minimally invasive (micro incision) otherwise for bone grafting area. Incisions must go through periosteum, allowing blade to reach the alveolar bone. A Piezo surgical knife (BS 1 insert, Piezotome ${ }^{\mathrm{TM}}$, Satelec Acteon Group, Merignac, France) is used to create cortical alveolar incision through the gingival micro-opening to a depth of approximately $3 \mathrm{~mm}$. Piezocision are finished, areas which requiring bone or soft tissue augmentations are tunneled using a small periosteal elevator through vertical incisions followed by grafting in the tunneled areas. Incisions are then closed using a resorbable 5-0 suture. Areas that have not been "tunneled" do not need suturing. After that, patient is prescribed with antibiotic and analgesic [5,6,12,13].

Adult treatment is often limited by clinician's ability to move teeth without the aid of active growth. Combined with a proper treatment planning and a good understanding of the biological events involved, piezocision technique can locally manipulate alveolar bone metabolism in order to obtain rapid and stable orthodontic results.

\section{REFERENCES}

[1] S. Aksakallia, B. Calikb, B. Karab, S. Ezirganli, “Accelerated tooth movement with piezocision and its periodontal-transversa effects in patients with class II malocclusion," Angle Orthod., vol. 86 (1), pp. 59-66, 2016

[2] O. Aylıkc1, C. Sakin, "Piezocision-assisted canine distalization,' J. Orthod. Res., vol. 1(2), pp.70-76, 2013.

[3] A. Domínguez, S.A. Velásquez, "Dental movement acceleration: literature review by an alternative scientific evidence method," World J. Methodol., vol. 4(3), pp. 151-162, 2014.

[4] E.I. Keser, S. Dibart, "Sequential piezocision: A novel approach to accelerated orthodontic treatment," Am. J. Orthod Dentofacial Ortho, vol. 144, pp. 879-889, 2013.

[5] S. Dibart, "Piezocision assisted orthodontic: past, present and future," Semin. Orthod., vol. 21(3), pp.170-175, 2015.

[6] J.D.M. Sebaoun, J. Surmenian, S. Dibart, "Accelerated orthodontic treatments with piezocision: a mini-invasive alternative to alveolar corticotomies," Orthod. Fr., vol. 82, pp. 311-319, 2011.

[7] S. Dibart, "Tissue response during piezocision-assisted tooth movement: a histological study in rats, ” Eur. J. Orthod., vol. 36 pp. 457-464, 2014

[8] H. Huang, R.C. Williams, S. Kyrkanides, "Accelerated orthodontic tooth movement: molecular mechanisms," Am. J. Orthod. Dentofacial Orthop., vol. 146(5), pp. 620-632, 2014

[9] M. Nishimura, M. Chiba, T. Ohashi, M. Sato, Y. Shimizu, K. Igarashi, "Periodontal tissue activation by vibration: intermittent stimulation by resonance vibration accelerates experimenta tooth movement in rat," Am. J. Orthod. Dentofacial Orthop. vol. 133, pp. 572-583, 2008.

[10] D.J. Ferguson, W.M. Wilcko, T.M. Wilcko, "Accelerating orthodontics by altering alveolar bone density," Good Practice, vol. 2, pp. 2-4, 2001.

[11] K. Cho, "The effect of cortical activation on orthodontic tooth movement," Oral Dis., vol. 13, pp. 314-319, 2007

[12] S. Dibart, J.D. Sebaoun, J. Surmenian, "Piezocision: minimally invasive, periodontally accelerated orthodontic tooth movement procedur," Compend. Contin. Educ. Dent., vol. 30(6), pp. 342-350, 2009

[13] P.E. Clínicas, "Rapid orthodontics with flapless piezoelectric corticotomies: first clinical experiences," Int. J. Odontostomat., vol. 7(1), pp. 79-85, 2013.

[14] D. Nelson, S. Dibart, "Sequential piezocision in a challenging adult case,” J. Clin. Orthod., vol. 48(9), pp. 555-562, 2014. 